\title{
Vibration-mediated correlation effects in the transport properties of a benzene molecule
}

\author{
Michael Knap, Enrico Arrigoni, and Wolfgang von der Linden \\ Institute of Theoretical and Computational Physics, Graz University of Technology, 8010 Graz, Austria
}

(Received 7 November 2012; published 7 August 2013)

\begin{abstract}
We theoretically analyze correlation effects on the transport properties of a benzene molecule that are mediated by interactions between the motion of the nuclei and the transmitted charge. We focus on the lowest-lying molecular vibrational mode which allows us to derive an analytic expression for the current. The results provide transparent interpretations of various features of the highly nonlinear current-voltage characteristics, which is experimentally accessible through resonant inelastic electron-tunneling spectroscopy.
\end{abstract}

DOI: 10.1103/PhysRevB.88.054301

PACS number(s): 73.63.-b, 63.22.-m, 73.23.Hk, 85.65.+h

\section{INTRODUCTION}

Vibrations of atoms in molecules or solids play an important role for many intriguing phenomena in various fields of physics. In condensed-matter novel states often emerge from phonon-mediated collective behavior. Among them are conventional superconductivity, ${ }^{1}$ colossal magnetoresistance ${ }^{2}$ and thermoelectricity. ${ }^{3}$ Localized vibrational modes are also believed to stabilize the $\alpha$ helix in proteins and to be responsible for storage and transport processes. ${ }^{4}$ In molecular electronics, the electron-phonon interactions strongly influence the conduction properties, contribute significantly to heating, and lead to nonlinear effects, including bistability, negative differential conductance, and hysteretic behavior. ${ }^{5-16}$ In these systems signatures of the interaction between charge carriers and molecular vibrations can be faithfully characterized by (resonant) inelastic electron tunneling [(R)IET] spectroscopy. ${ }^{15,17,18}$ The importance of vibrational modes on the conduction properties of molecular transistors has been demonstrated in various seminal experiments. ${ }^{19-26}$

A wide range of theoretical studies concerning nonequilibrium properties of nanoscale devices are based on the nonequilibrium Green's function (NEGF) method evaluated in the frame of the local density approximation (LDA) (see, e.g., Refs. 27 and 28 and references therein). On one hand, the LDA-NEGF approach is a first-principles approach and as such makes it possible to study realistic devices, on the other hand, correlation effects, irrespective of whether they are of electron-electron or electron-phonon character, are included only to low order. This can lead to unphysical results provided the interaction is strong. ${ }^{29}$

In the theoretical approach presented here, we start out with the exact expression for the current within the NEGF framework and employ a strong-coupling cluster approximation to evaluate the required Green's functions (GFs). ${ }^{30,31}$ This allows exactly taking into account all interactions of the nanoscale device itself, be it of electron-electron or electron-phonon nature, while treating the influence of the leads perturbatively. As an application, we investigate the nonequilibrium transport across a benzene molecule [Fig. 1(a)] and observe that,

Published by the American Physical Society under the terms of the Creative Commons Attribution 3.0 License. Further distribution of this work must maintain attribution to the author(s) and the published article's title, journal citation, and DOI. at low temperatures, phonon-mediated interactions leave a vibrational fingerprint qualitatively similar to those observed in (R)IET spectroscopy. ${ }^{19-26}$

At low temperatures we obtain a stepwise increase in the current at voltage differences given by twice the phonon energy, Fig. 1(b). This stepwise increase manifests in the conductance and in the differential shot noise through multiple equally spaced peaks, Figs. 1(c) and 1(d) as well as in a branching of the diamond structure in the stability diagram, Fig. 3. At low bias voltage the current is significantly suppressed due to electron-phonon interactions, which is reminiscent of the Franck-Condon blockade in molecular devices, ${ }^{6}$ see the inset of Fig. 1(b).

\section{THEORETICAL DESCRIPTION}

We consider a single benzene aromatic ring coupled to metallic leads, Fig. 1(a). The benzene molecule is modeled by the Hamiltonian,

$$
H_{b}=H_{e}+H_{p}+H_{e p} .
$$

The first term describes the motion of the electrons,

$$
H_{e}=\sum_{i, \sigma}\left[-t\left(c_{i \sigma}^{\dagger} c_{i+1 \sigma}+\text { H.c. }\right)+\epsilon c_{i \sigma}^{\dagger} c_{i \sigma}\right],
$$

where $t$ is the hopping amplitude and $\epsilon$ is the local energy, which can be controlled by the gate voltage $V_{g}$, i.e., $\epsilon \rightarrow \epsilon+$ $e V_{g}$. Electrons with spin $\sigma$ on orbital $i$ are created (destroyed) by $c_{i \sigma}^{\dagger}\left(c_{i \sigma}\right)$. In this paper, we consider an effective 12 spinorbital model, describing the delocalized $\pi$ bonds of benzene. The second term $H_{p}$ is the bare vibrational part, which we transform into the eigenmodes,

$$
H_{p}=\sum_{m} \hbar \omega_{m} b_{m}^{\dagger} b_{m}
$$

where $b_{m}^{\dagger}\left(b_{m}\right)$ create (destroy) phonons in mode $m$ with energy $\hbar \omega_{m}$. The last term of (1) incorporates the Su-SchriefferHeeger $^{32}$ interaction between the electrons and the dynamic phonons

$$
H_{e p}=\sum_{m j \sigma} g_{m}\left(b_{m}^{\dagger}+b_{m}\right)\left(D_{m}^{(j, j+1)} c_{j \sigma}^{\dagger} c_{j+1 \sigma}+\text { H.c. }\right),
$$

with $g_{m}:=g \sqrt{\hbar / 2 M \omega_{m}}, g$ as the electron-phonon interaction, $M$ as the mass of the nuclei, and $D_{m}$ as the displacement 


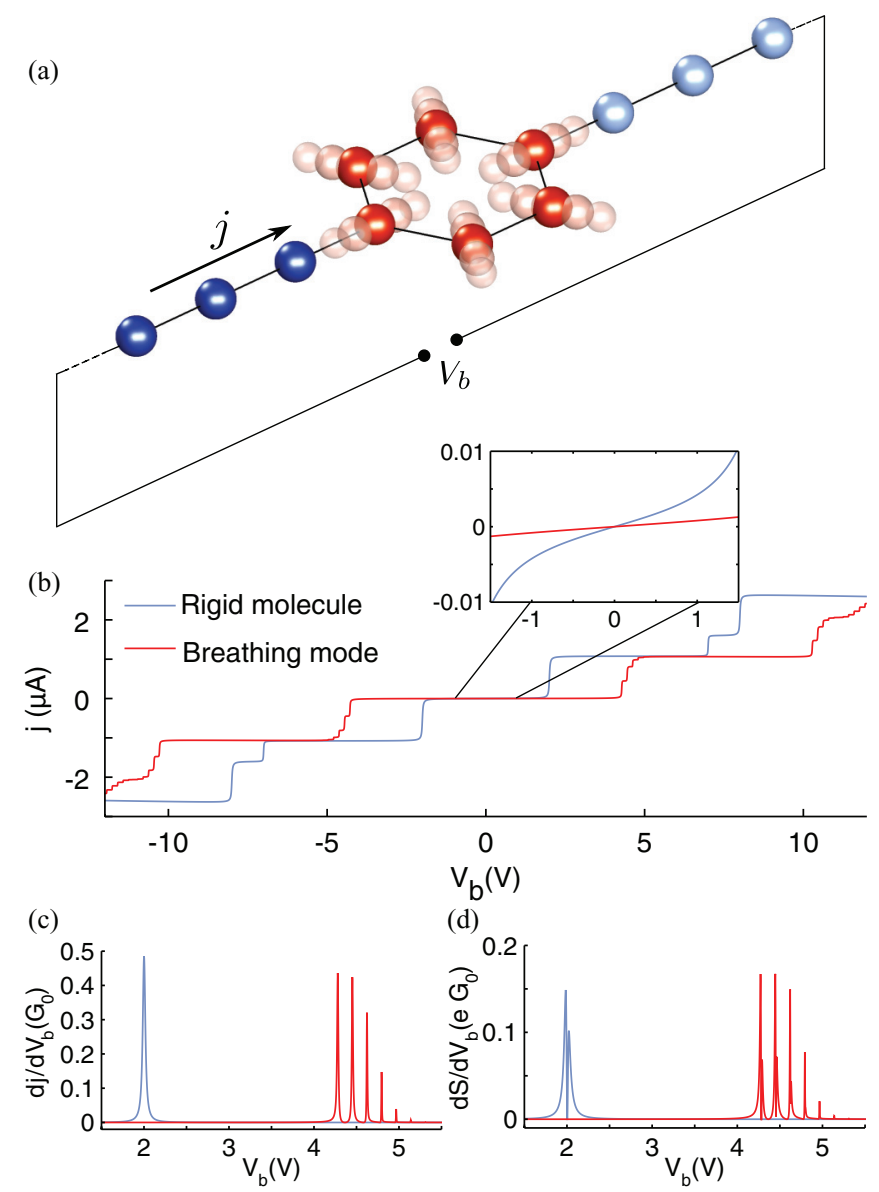

FIG. 1. (Color online) (a) Illustration of the phonon breathing mode in a benzene molecule (red spheres), which is coupled to metallic leads (blue spheres). (b) The bias voltage $V_{b}$ induces a current $j$ that is significantly modified by the interaction between the nuclear motion and the charge. At low $V_{b}$ the current is suppressed, ${ }^{6}$ see the inset, whereas, at large $V_{b}$ strong nonlinear behavior leads to a stepwise increase in the current, also manifesting in (c) the conductance and (d) the differential shot noise through multiple equally spaced peaks. The data are evaluated at $T=10 \mathrm{~K}$.

matrix of eigenmode $m$, which is given by

$$
D_{m}^{(j, j+1)}=\frac{1}{\sqrt{6}} e^{i(\pi / 3) m j} \quad \text { with } \quad m \in\{0,1, \ldots, 5\} .
$$

The metallic leads are described by semi-infinite tight-binding chains with hopping $t_{\alpha}$, on-site energy $\epsilon_{\alpha}$, and chemical potential $\mu_{\alpha}$ ( $\alpha=$ left or right). We assume that the bias voltage $e V_{b}=\mu_{l}-\mu_{r}$ is applied symmetrically, i.e., $\mu_{l}=$ $\epsilon_{l}=-\mu_{r}=-\epsilon_{r}$ and that the leads are equilibrated, i.e., that the occupation follows the Fermi-Dirac statistics $f_{\alpha}=$ $\left[e^{\left(\varepsilon-\mu_{\alpha}\right) / k_{B} T}+1\right]^{-1}$, where $k_{B}$ is the Boltzmann constant and $T$ is the temperature.

In this paper, we use the NEGF framework to evaluate the nonequilibrium steady-state properties along the lines presented in Ref. 30. Due to the electron-phonon interaction it is, in general, not possible to solve the problem exactly, and thus, we employ cluster perturbation theory (CPT): ${ }^{33}$ In short, the system is first divided into several clusters, each of which can be solved exactly either analytically or numerically, taking correlations on the cluster level into account exactly. Strong coupling CPT is then employed to connect the individual clusters.

Here, we divide the molecular device into three clusters: (i) the central region consisting of the benzene ring with vibrational degrees of freedom, (ii) the left, and (iii) the right metallic leads, which are connected to the molecular device. From the exact solution of the isolated interacting central region, we obtain an approximate self-energy of the full system. The results can also be systematically improved by incorporating lead sites into the central system. ${ }^{30,31}$ Based on considerations similar to those of Meir and Wingreen, ${ }^{34}$ we can show that within this CPT approach the current is of the Landauer-Büttiker form, ${ }^{35,36}$ even though correlations are included exactly on the cluster level. In particular, we find

$$
j=\frac{e}{\hbar} \int \frac{d \varepsilon}{2 \pi}\left(f_{l}-f_{r}\right) \operatorname{Tr}[\mathcal{T}],
$$

where $\mathcal{T}$ is the transmission coefficient matrix,

$$
\mathcal{T}:=\mathcal{G}^{R}(\varepsilon) \Gamma_{r}(\varepsilon) \mathcal{G}^{A}(\varepsilon) \Gamma_{l}(\varepsilon) .
$$

This matrix contains the retarded (advanced) $\mathrm{GF} \mathcal{G}^{R}\left[\mathcal{G}^{A}=\right.$ $\left.\left(\mathcal{G}^{R}\right)^{\dagger}\right]$, which within the CPT is given by $\left(\mathcal{G}^{R}\right)^{-1}=\left(g^{R}\right)^{-1}-$ $\left(\tilde{\Sigma}_{l}+\tilde{\Sigma}_{r}\right)$, where $g^{R}$ is the exact retarded GF of the isolated benzene molecule, $\tilde{\Sigma}_{\alpha}:=\mathrm{T}_{c \alpha} g_{\alpha \alpha}^{R} \mathrm{~T}_{\alpha c}$ takes into account the effect of lead $\alpha$ on the GF, $\mathrm{T}_{c \alpha}$ is the tunneling coupling between the central device and the lead $\alpha$, and $g_{\alpha \alpha}^{R}$ stands for the retarded lead $\mathrm{GF}^{30}$ In addition, the transmission $\mathcal{T}$ depends on the imaginary part of the lead-induced self-energy via $\Gamma_{\alpha}=-2 \operatorname{Im} \tilde{\Sigma}_{\alpha}$. Similarly, the expression for the shot noise, i.e., current-current correlations, in the zero-frequency limit reads ${ }^{37}$

$$
\begin{aligned}
S(\omega \rightarrow 0):=S & =\frac{e^{2}}{\hbar} \int \frac{d \varepsilon}{2 \pi}\left[f_{l}\left(1-f_{l}\right)+f_{r}\left(1-f_{r}\right)\right] \operatorname{Tr}[\mathcal{T}] \\
& +\left(f_{l}-f_{r}\right)^{2} \operatorname{Tr}[(1-\mathcal{T}) \mathcal{T}] .
\end{aligned}
$$

In principle the nonequilibrium properties can be determined by solving the eigenvalue problem of $H_{b}$ numerically taking all vibrational modes into account using the formulas discussed above. Within this approach it is also straightforward to introduce electron-electron interactions giving rise to Kondo physics provided the molecule is (asymmetrically) electron or hole doped. ${ }^{38}$

In the present paper, however, we want to study the main effects generated by the electron-phonon interaction. In order to gain insight into the problem, we focus on a single vibrational mode, in particular, on the breathing mode illustrated in Fig. 1(a). Coupling the benzene ring to the leads breaks the $\mathrm{C} 6$ rotational invariance of the molecule. However, calculations reveal that a breathinglike mode should still exist in that case. A very appealing feature of this somewhat simplified mode is the fact that the resulting interacting model of the central region can be solved analytically and thus provides a transparent insight into phonon-mediated correlation effects on the nonequilibrium physics of such a molecular electronic device.

\section{ANALYTIC SOLUTION}

For a single vibrational mode $\mu$ (not necessarily the breathing mode) we can readily eliminate the electron-phonon 

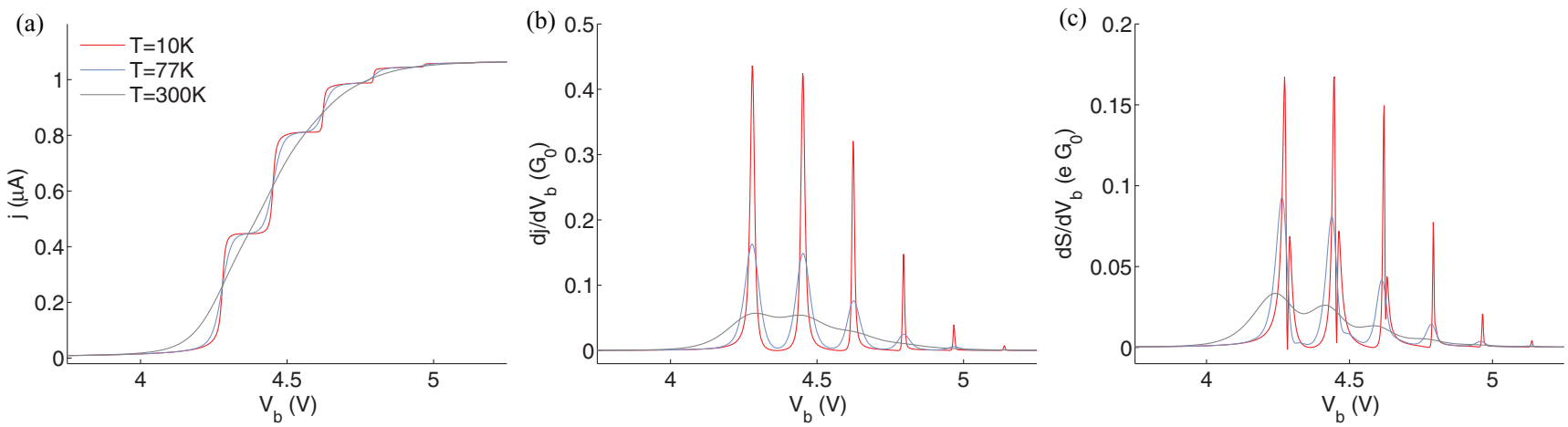

FIG. 2. (Color online) Temperature dependence of (a) the current, (b) the conductance, and (c) the differential shot noise versus bias voltage $V_{b}$ curves. At temperatures $T$ much lower than the phonon energy $\omega_{\mu}=0.086 \mathrm{eV}$, which is the smallest energy scale in Eq. (1), the phonon-mediated steps and peaks, respectively, are pronounced and clearly visible. For increasing temperature these features are washed out, and at room temperature $T=300 \mathrm{~K}$, hardly any remain.

interaction by a unitary transformation of the Lang-Firsov type. ${ }^{28}$ To this end we first diagonalize the displacement matrix $D_{\mu} X=X \Lambda, \Lambda$ is the diagonal matrix with the eigenvalues, and $X$ is the corresponding matrix of the eigenvectors and transforms the electron operators accordingly, $\mathbf{d}=X \mathbf{c}$ where we introduce the vector notation $\mathbf{c}^{\dagger}=\left(c_{1 \uparrow}^{\dagger}, c_{2 \uparrow}^{\dagger}, \ldots, c_{L \downarrow}^{\dagger}\right)$. The Lang-Firsov-like unitary transformation is generated by the operator,

$$
e^{S}:=e^{\left(g_{\mu} / \hbar \omega_{\mu}\right)\left(b-b^{\dagger}\right) \mathbf{d}^{\dagger} \Lambda \mathbf{d}} .
$$

All operators that are subject to the unitary transformation are denoted by $\bar{O}:=e^{S} O e^{-S}$, which, in particular, results in

$$
\bar{d}_{k \sigma}^{\dagger}=d_{k \sigma}^{\dagger} e^{\left(g_{\mu} / \hbar \omega_{\mu}\right)\left(b-b^{\dagger}\right) \lambda_{k}} .
$$

The Lang-Firsov transformation eliminates the explicit electron-phonon interaction and results in an effective Hamiltonian $\bar{H}_{b}=\bar{H}_{e}+\hbar \omega_{\mu} b^{\dagger} b$. The electronic GF entering the transport properties of the molecular junction [Eq. (3)] is transformed accordingly, i.e., $g(k, \omega) \rightarrow \bar{g}(k, \omega)$ by replacing $d_{k \sigma}^{\dagger} \rightarrow \bar{d}_{k \sigma}^{\dagger}$ in its definition. For the particular case of the breathing mode, the matrices $D_{\mu}$ and the matrix describing the nearest-neighbor hopping commute and, thus, can be diagonalized by a common set of eigenvectors. In this case we find $\lambda_{k}=\sqrt{2 / 3} \cos k$ and $X_{n k}=\exp (i k n) / \sqrt{6}$. The $d_{k}^{\dagger}$, s, thus, create electrons with a given (angular) momentum $k$ in the molecule. In the transformed Hamiltonian the electronic part becomes

$$
\bar{H}_{e}=\sum_{k \sigma}(\epsilon-2 t \cos k) \bar{b}_{k \sigma}^{\dagger} \bar{b}_{k \sigma}-U\left(\sum_{k \sigma} \bar{b}_{k \sigma}^{\dagger} \bar{b}_{k \sigma} \cos k\right)^{2} .
$$

After this transformation, the electron and vibrational degrees of freedom are completely decoupled but, instead, an attractive electron-electron interaction proportional to $U:=\frac{2 g_{\mu}^{2}}{3 \hbar \omega_{\mu}}$ emerges. At first sight Hamiltonian (7) might be reminiscent of the Holstein Hamiltonian; note, however, that the parentheses enclose the sum in the second term, which gives rise to additional contributions mixing different angular momenta $k$.

The interacting Hamiltonian (7) can be solved exactly. The eigenvectors are of the form $|n\rangle\left|K_{\uparrow}\right\rangle\left|K_{\downarrow}\right\rangle$, i.e., they are tensor products of the oscillator eigenvectors $|n\rangle$, and the Slater determinants $\left|K_{\sigma}\right\rangle:=\prod_{k \in K_{\sigma}} \bar{d}_{k \sigma}^{\dagger}|0\rangle$ are composed of the molecular orbital operators $d_{k \sigma}^{\dagger}$ dressed by bond distortions that are encoded in the Lang-Firsov factor of Eq. (6). In Eq. (7) the dressed particles (polarons) are conserved, however, the current is calculated for the electrons, which exhibit inelastic electron-phonon scattering. For the electron GF of a single spin species, we find

$$
\begin{aligned}
\bar{g}(k, \omega)= & \sum_{n} \frac{e^{-\alpha_{k}^{2}} \alpha_{k}^{2 n}}{n !} \\
& \times\left[\frac{\Theta\left(k>k_{F}\right)}{\hbar \omega-\left(E_{k n}^{+}-E_{0}\right)}+\frac{\Theta\left(k<k_{F}\right)}{\hbar \omega+\left(E_{k n}^{-}-E_{0}\right)}\right],
\end{aligned}
$$

with $\alpha_{k}=\sqrt{U} \cos k$, and the energies are

$$
\begin{aligned}
E_{0} & =\epsilon \mathcal{N}_{0}-2 t \mathcal{C}_{0}-U \mathcal{C}_{0}^{2}, \\
E_{k n}^{ \pm} & =n \hbar \omega_{\mu}+\left(\mathcal{C}_{0} \pm 1\right) \epsilon-2 t\left(\mathcal{C}_{0} \pm \cos k\right)-U\left(\mathcal{C}_{0} \pm \cos k\right)^{2}, \\
\mathcal{C}_{0} & =\sum_{\sigma} \sum_{q \in \mathrm{FS}} \cos q .
\end{aligned}
$$

$\mathcal{N}_{0}$ is the equilibrium number of electrons in the benzene molecule, and FS denotes the Fermi sea. The physical properties of the system are particularly transparent in the differential conductance, which up to order $O\left(V_{b}^{2} / T^{2}\right)$ and $O\left(t^{\prime 2} / t_{\alpha}^{2}\right)$ is given by

$$
\frac{d j}{d V_{b}}=\frac{e}{4 \hbar \pi}\left[\mathcal{T}\left(V_{b} / 2\right)+\mathcal{T}\left(-V_{b} / 2\right)\right] .
$$

Along with Eqs. (3) and (8), it can be deduced that current pathways open up whenever $V_{b} / 2$ is equal to the excitation energy $\hbar \omega=E_{k n}^{+}+\hbar \omega_{\mu} n$ in the particle sector or $\hbar \omega=$ $-E_{k n}^{-}+\hbar \omega_{\mu} n$ in the hole sector.

\section{TRANSPORT PROPERTIES}

Next, the impact of phonons on the transport properties will be investigated guided by the breathing mode of a benzene molecule. We use the parameters reported in Refs. 13 and 39 

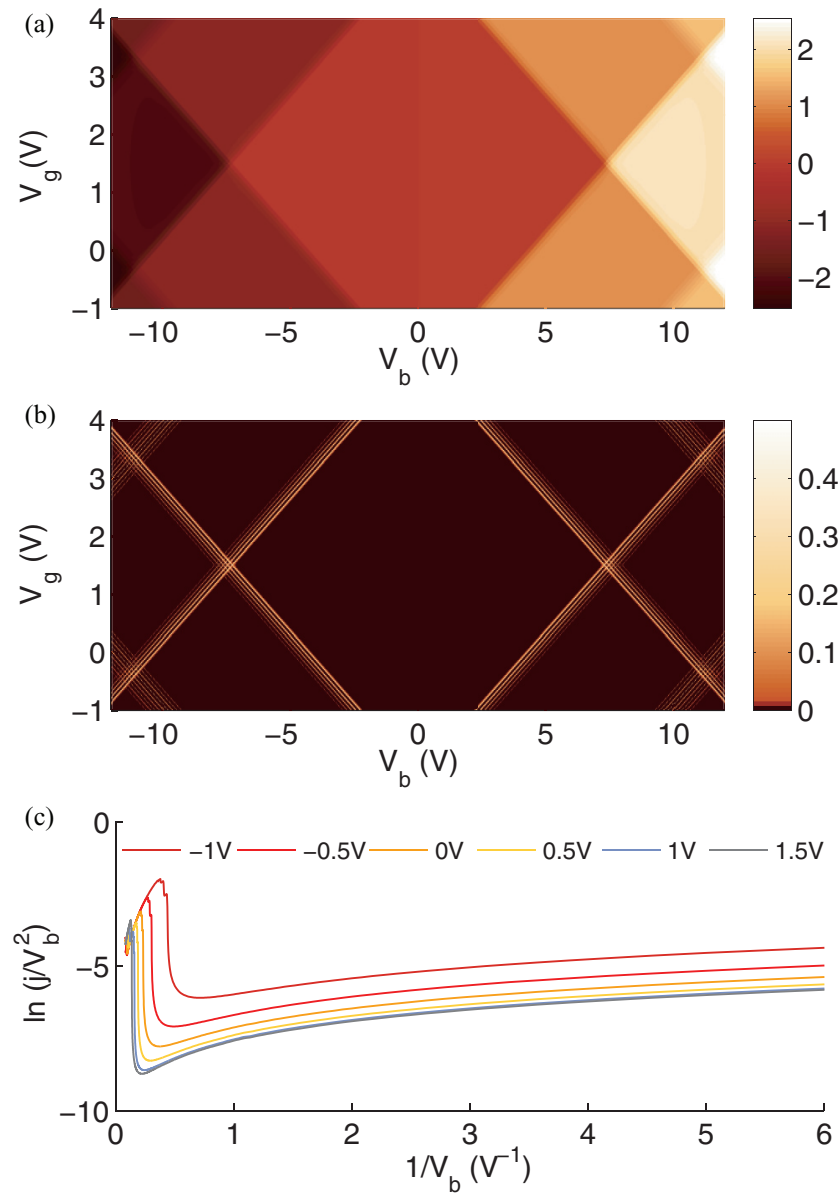

FIG. 3. (Color online) (a) Current as a function of the bias voltage $V_{b}$ and the gate voltage $V_{g}$ and (b) corresponding stability diagram (i.e., differential conductance). The electron-phonon interaction increases the area of the central diamond and induces a splitting of the lines in the stability diagram. (c) Fowler-Nordheim plots for a few selected values of the gate voltage $V_{g}$ specified in the legend. Data evaluated for $T=10 \mathrm{~K}$.

for the benzene molecule and the electron-phonon coupling; in particular, we set $t=2.5, \epsilon=-1.5, \hbar \omega_{\mu}=\sqrt{K / M}=$ 0.086 , and $g_{\mu}=0.18 \mathrm{eV}$. In addition, for the metallic leads we choose $t_{\alpha}=12 \mathrm{eV}$ close to the wideband limit, and the coupling between the leads and the benzene ring is $t^{\prime}=0.4 \mathrm{eV}$. Therefore, the smallest energy scale in the model is the phonon energy $\hbar \omega_{\mu}$. The current evaluated at $T=10 \mathrm{~K}$ (the temperature enters through the Fermi-Dirac distribution functions of the leads) for the rigid molecule as well as the molecule with the breathing mode is shown in Fig. 1(b). At low bias voltage, we observe a suppression of the current due to electron-phonon interactions, reminiscent of the Franck-Condon blockade, ${ }^{6}$ see the inset. Due to the electron-phonon-mediated correlations [second term in Eq. (7)], the gap in the density of states of the benzene molecule widens considerably. Consequently, the threshold for the current increases to $V_{b} \sim 4 \mathrm{eV}$. Above the threshold the current increases stepwise at voltage differences given by twice the phonon energy $\hbar \omega_{\mu}$, until the next plateau is reached. The new current pathways are related to the vibrational excitations, and the factor 2 is due to the argument $V_{b} / 2$ in Eq. (9).
This also manifests in the differential conductance Fig. 1(c) and in the differential shot noise Fig. 1(d) through pronounced equally spaced peaks. At the maxima of the conductance, the differential shot noise, corresponding to the derivative of the current-current correlation for $\omega \rightarrow 0$, exhibits dips. ${ }^{37}$ At low temperatures this is observable for a few low-energy peaks of the phonon branching. A detailed analysis of the differential conductance shows that the peaks are near the poles of the cluster GF $\bar{g}$ of the decoupled device. This is reasonable since the effective coupling $V / t^{\prime 2}$ is small. Therefore, the impact of $\tilde{\Sigma}_{\alpha}$ stems primarily from its imaginary part which determines the width and the weight of the individual peaks. So the measurement of these quantities can be used as a diagnostic tool to analyze the device and to extract information about the electronic and phononic excitations. In particular, the equidistant peaks in the conductance and the tips in the differential shot noise are a fingerprint of the bare vibrational excitations. One should notice, however, that for general vibrational modes the distance between the peaks does not need to be equidistant since the electronic excitations will depend on the phonon occupation $n$ due to $\bar{H}_{e}$.

For increasing temperature the vibrational features are smeared out since the phonon energy $\omega_{\mu}=86 \mathrm{meV}$ is rather small and comparable to room temperature $T=300 \mathrm{~K} \sim$ $26 \mathrm{meV}$. In Fig. 2, the current, the differential conductance, and the differential shot noise are shown at different temperatures $T=\{10,77,300\} \mathrm{K}$. With increasing temperature the steps in the current are smoothed out, and the peaks in the conductance and the differential shot noise decrease significantly. In addition, the dips in the shot noise vanish.

Finally, we study the consequence of orbital gating where the local on-site energy of the benzene molecule is changed by a gate voltage. Experimentally orbital gating has recently been achieved in Ref. 26. The current as a function of the gate voltage $V_{g}$ and the bias voltage $V_{b}$ exhibits the celebrated diamond structure centered around the particle-hole symmetric point $V_{g}=1.5 \mathrm{~V}$, Fig. 3(a). As compared to the rigid molecule, see Figs. 1(b)-1(d), the area of the center diamond is increased due to the electron-phonon interactions, and the transition between the diamonds involves several steps, best visible in the stability diagram, corresponding to the conductance in the $V_{g}-V_{b}$ plane, shown in Fig. 3(b). The Fowler-Nordheim plots (c) which show $\ln \left(j / V_{b}^{2}\right)$ as a function of $V_{b}^{-1}$ compare well with the experimental observation in Ref. 26. From these plots different tunneling regimes become apparent: At low bias voltage, the "logarithmic" dependence indicates direct tunneling, whereas, for increasing bias voltage the curve has a large negative slope, indicating field emission. ${ }^{26}$ Close to this bias voltage phonon-mediated interactions manifest as small wiggles in the curves. When further increasing the voltage, the next plateau is reached, which is accompanied by a reentrance in the direct tunneling regime. Similar to Ref. 26 the minimum of the curves moves to larger bias voltages for increasing gate voltage reflecting, the diamond shape of Figs. 3(a) and 3(b).

\section{CONCLUSIONS AND OUTLOOK}

To summarize, we explored phonon-mediated correlation effects on the transport properties of a single benzene molecule. We focused on an analytically tractable single-mode 
model that reveals a deeper understanding of the impact of phonons on experimental (R)IET spectra. The theoretical approach we presented makes it possible to determine nonequilibrium properties of strongly correlated nanoscale devices. When solving the interacting device totally numerically, other vibrational modes and electron-electron interactions can be included as well.

\section{ACKNOWLEDGMENTS}

We are grateful to G. Cuniberti, R. Gutiérrez, and D. A. Ryndyk for insightful discussions. The authors acknowledge support from the Austrian Science Fund (FWF) under Projects No. P24081-N16 and No. J 3361-N20. Calculations have been performed on the Vienna Scientific Cluster (VSC II).
${ }^{1}$ J. Bardeen, L. N. Cooper, and J. R. Schrieffer, Phys. Rev. 108, 1175 (1957).

${ }^{2}$ A. J. Millis, Philos. Trans. R. Soc. London, Ser. A 356, 1473 (1998).

${ }^{3}$ O. Delaire, J. Ma, K. Marty, A. F. May, M. A. McGuire, M.-H. Du, D. J. Singh, A. Podlesnyak, G. Ehlers, M. D. Lumsden, and B. C. Sales, Nat. Mater. 10, 614 (2011).

${ }^{4}$ A. Scott, Phys. Rep. 217, 1 (1992).

${ }^{5}$ K. Flensberg, Phys. Rev. B 68, 205323 (2003).

${ }^{6}$ J. Koch and F. von Oppen, Phys. Rev. Lett. 94, 206804 (2005).

${ }^{7}$ M. Galperin, A. Nitzan, and M. A. Ratner, Phys. Rev. B 73, 045314 (2006).

${ }^{8}$ D. A. Ryndyk, M. Hartung, and G. Cuniberti, Phys. Rev. B 73, 045420 (2006).

${ }^{9}$ C. Benesch, M. Č́žžek, M. Thoss, and W. Domcke, Chem. Phys. Lett. 430, 355 (2006).

${ }^{10}$ D. A. Ryndyk and G. Cuniberti, Phys. Rev. B 76, 155430 (2007).

${ }^{11}$ M. Galperin, M. A. Ratner, and A. Nitzan, J. Phys.: Condens. Matter 19, 103201 (2007).

${ }^{12}$ M. Galperin, M. A. Ratner, A. Nitzan, and A. Troisi, Science 319, 1056 (2008).

${ }^{13}$ A. L. Botelho, Y. Shin, M. Li, L. Jiang, and X. Lin, J. Phys.: Condens. Matter 23, 455501 (2011).

${ }^{14}$ G. Cuniberti, K. Richter, and G. Fagas, Introducing Molecular Electronics, 1st ed. (Springer, Heidelberg, Germany, 2005).

${ }^{15}$ J. C. Cuevas and E. Scheer, Molecular Electronics: An Introduction to Theory and Experiment (Nanotechnology and Nanoscience), 1st ed. (World Scientific, Singapore, 2010).

${ }^{16}$ H. Song, M. A. Reed, and T. Lee, Adv. Mater. 23, 1583 (2011).

${ }^{17}$ R. C. Jaklevic and J. Lambe, Phys. Rev. Lett. 17, 1139 (1966).

${ }^{18}$ B. C. Stipe, M. A. Rezaei, and W. Ho, Science 280, 1732 (1998).

${ }^{19}$ H. Park, J. Park, A. K. L. Lim, E. H. Anderson, A. P. Alivisatos, and P. L. McEuen, Nature (London) 407, 57 (2000).

${ }^{20}$ N. B. Zhitenev, H. Meng, and Z. Bao, Phys. Rev. Lett. 88, 226801 (2002).

${ }^{21}$ R. H. M. Smit, Y. Noat, C. Untiedt, N. D. Lang, M. C. van Hemert, and J. M. van Ruitenbeek, Nature (London) 419, 906 (2002).
${ }^{22}$ X. H. Qiu, G. V. Nazin, and W. Ho, Phys. Rev. Lett. 92, 206102 (2004).

${ }^{23}$ A. N. Pasupathy, J. Park, C. Chang, A. V. Soldatov, S. Lebedkin, R. C. Bialczak, J. E. Grose, L. A. K. Donev, J. P. Sethna, D. C. Ralph, and P. L. McEuen, Nano Lett. 5, 203 (2005).

${ }^{24}$ O. Tal, M. Krieger, B. Leerink, and J. M. van Ruitenbeek, Phys. Rev. Lett. 100, 196804 (2008).

${ }^{25}$ M. Kiguchi, O. Tal, S. Wohlthat, F. Pauly, M. Krieger, D. Djukic, J. C. Cuevas, and J. M. van Ruitenbeek, Phys. Rev. Lett. 101, 046801 (2008).

${ }^{26}$ H. Song, Y. Kim, Y. H. Jang, H. Jeong, M. A. Reed, and T. Lee, Nature (London) 462, 1039 (2009).

${ }^{27}$ T. Frederiksen, M. Paulsson, M. Brandbyge, and A.-P. Jauho, Phys. Rev. B 75, 205413 (2007).

${ }^{28}$ D. A. Ryndyk, R. Gutiérrez, B. Song, and G. Cuniberti, in Energy Transfer Dynamics in Biomaterial Systems, edited by I. Burghardt, V. May, D. A. Micha, E. R. Bittner, A. W. Castleman, J. P. Toennies, K. Yamanouchi, and W. Zinth, Springer Series in Chemical Physics Vol. 93 (Springer, Berlin, Heidelberg, 2009), p. 213.

${ }^{29}$ W. Lee, N. Jean, and S. Sanvito, Phys. Rev. B 79, 085120 (2009).

${ }^{30}$ M. Knap, W. von der Linden, and E. Arrigoni, Phys. Rev. B 84, 115145 (2011).

${ }^{31}$ M. Nuss, C. Heil, M. Ganahl, M. Knap, H. G. Evertz, E. Arrigoni, and W. von der Linden, Phys. Rev. B 86, 245119 (2012).

${ }^{32}$ A. J. Heeger, S. Kivelson, J. R. Schrieffer, and W. P. Su, Rev. Mod. Phys. 60, 781 (1988).

${ }^{33}$ D. Sénéchal, D. Perez, and M. Pioro-Ladrière, Phys. Rev. Lett. 84, 522 (2000).

${ }^{34}$ Y. Meir and N. S. Wingreen, Phys. Rev. Lett. 68, 2512 (1992).

${ }^{35}$ R. Landauer, Philos. Mag. 21, 863 (1970).

${ }^{36}$ M. Büttiker, Phys. Rev. Lett. 57, 1761 (1986).

${ }^{37}$ J.-X. Zhu and A. V. Balatsky, Phys. Rev. B 67, 165326 (2003).

${ }^{38}$ C. Romeike, M. R. Wegewijs, W. Hofstetter, and H. Schoeller, Phys. Rev. Lett. 96, 196601 (2006).

${ }^{39}$ D. Rai, O. Hod, and A. Nitzan, J. Phys. Chem. C 114, 20583 (2010). 